\title{
O PET-SAÚDE COMO NORTEADOR DA FORMAÇÃO EM ENFERMAGEM PARA O SISTEMA ÚNICO DE SAÚDE
}

\author{
PET-HEALTH AS A GUIDE FOR THE TRAINING IN \\ NURSING FOR THE UNIFIED HEALTH SYSTEM
}

\author{
Luiz Roberto Augusto Noro ${ }^{1}$, José Luis Medina Moya ${ }^{2}$ \\ ${ }^{1}$ Universidade Federal do Rio Grande do Norte, Centro de Ciências da Saúde, Natal, Rio Grande do \\ Norte, Brasil. \\ <luiznoro@ccs.ufrn.br> \\ ${ }^{2}$ Universidade de Barcelona, Faculdade de Educação, Departamento de Ensino e Organização Educa- \\ cional, Barcelona, Espanha.
}

Resumo A saúde de qualidade e de acesso universal é um direito que deve ser garantido por políticas públicas, financiamento compatível e pela adequada formação dos profissionais de saúde. Uma das estratégias identificadas para alcançar esse desafio é o Programa de Educação para o Trabalho em Saúde, que visa promover a interação ativa de alunos e professores com os serviços de saúde. O objetivo deste estudo foi comparar, por meio dos resultados do Exame Nacional de Desempenho dos Estudantes dos cursos de Enfermagem de 2013, o desempenho dos que participaram com o daqueles que não fizeram parte do referido Programa. Trata-se de estudo observacional, de corte transversal analítico, que analisou o desempenho pelas médias ponderadas em formação geral, formação profissional e em Saúde Coletiva. A pesquisa analisou o desempenho pelas médias ponderadas em formação geral, formação profissional e saúde coletiva. A amostra do estudo consistiu de 30.289 estudantes, dos quais 876 vinculados ao Programa de Educação para o Trabalho em Saúde. Os alunos envolvidos com o Programa tiveram um desempenho superior em todos os segmentos, com diferença estatisticamente significativa ( $\mathrm{p}<0,001)$ em comparação com aqueles que não participaram. Os resultados indicam que o Programa em questão tem potência para compreender a necessidade da formação no Sistema Único de Saúde, ao envolver conhecimentos de Saúde Coletiva e da área profissional, e deve ser elemento norteador da construção dos currículos dos cursos de Enfermagem.

Palavras-chave Sistema Único de Saúde; avaliação educacional; enfermagem.
Abstract Quality and universal health care is a right that should be guaranteed through public policies, proper funding, and through the adequate training of the health professionals. One of the strategies identified to overcome this challenge is the Education for the Work in Health Program (Programa de Educação para o Trabalho em Saúde, PET-Saúde, in the Portuguese acronym; PET-Health henceforth), which has the goal of promoting the interaction of students and professors with the health services. The goal of the present study was to compare, using the results of the 2013 National Nursing Student Performance Exam (Exame Nacional de Desempenho dos Estudantes dos cursos de Enfermagem, in Portuguese), the performance of the individuals who took part in the mentioned program with that of those who did not. It is an observational study, with a cross-sectional and analytical perspective, which was conducted between February and June 2017. The research analyzed the performance through the weighted arithmetic means regarding general training, professional training and Collective Health. The sample was composed of 30,289 students, 876 of which took part in the PET-Health. The students involved with the program performed better in every segment, with a statistically significant difference ( $p<0.001)$ in comparison to those who did not take part in the program. The results indicate that the program in question has the power to understand the need for training within the Unified Health System (Sistema Único de Saúde, SUS, in the Portuguese acronym) regarding the knowledge on Collective Health and the professional area, which should be a guiding element in the development of the syllabuses of Nursing Courses.

Keywords Unified Health System; educational evaluation; nursing

Este é um artigo publicado em acesso aberto sob uma licença Creative Commons. 


\section{Introdução}

A saúde de qualidade e de acesso universal, segundo a constituição brasileira, é um direito fundamental, que deve ser garantido por políticas públicas, financiamento compatível e pela adequada formação dos profissionais de saúde. Políticas públicas dependem dos governantes e dos responsáveis pela execução de suas atribuições, considerando a formação que tiveram ao longo de suas vidas. Na formação de profissionais de saúde, segundo Pereira e Lages (2013), é fundamental que o Sistema Único de Saúde (SUS) seja compreendido como campo fundamental de aprendizagem, conforme preconizado em todas as Diretrizes Curriculares Nacionais (DCNs) dos cursos de graduação da área da saúde.

As DCNs de todos os cursos da área da saúde definem o perfil do egresso generalista, humanista, com visão crítica e reflexiva sobre seu papel na transformação da sociedade; preveem o desenvolvimento de competências e habilidades gerais voltadas para a atenção à saúde, educação permanente, liderança, administração e gerenciamento, comunicação e tomada de decisões e propõem a construção de um projeto pedagógico que respeite o aluno como sujeito de seu aprendizado. Além desses elementos, o que contribuiu para a efetiva articulação da formação com a área da saúde foi a exigência presente em todas as DCNs da área da saúde formação centrada no SUS, prerrogativa prevista desde a lei n. 8080, de 19 de setembro de 1990 (Brasil, 1990), mas nunca considerada para a maioria das instituições de educação superior (IESs).

Em estudo de Haddad (2011), observou-se que, entre as profissões da área da saúde, a enfermagem se destaca como uma das que mais se vincula à formação direcionada para o SUS, com enfoque especial na atenção primária e na gestão dos serviços.

A meta maior, sinalizada pelas DCNs dos cursos de enfermagem, aponta para a formação de profissionais de saúde sensíveis aos principais problemas sociais e sanitários da população. Tais diretrizes são pautadas por um currículo integrado, desenvolvido com metodologias ativas de aprendizagem, tendo como guia o direito universal à saúde (Winters, Prado e Heidemann, 2016).

Entretanto, é fundamental compreender a complexidade da formação em enfermagem, considerando ser um dos cursos, conforme sinalizado por Pierantoni e Magnago (2017), com maior número de vagas entre todos da área da saúde (178.264, em 2014). Mais de 75\% dessas vagas são em instituições privadas de educação superior. Não há dúvida, portanto, do papel que a formação em enfermagem carrega na determinação do SUS como cenário privilegiado de aprendizado para os futuros profissionais.

Uma das estratégias para fortalecer esse desafio é o Programa de Educação para o Trabalho em Saúde (PET-Saúde), implantado desde 2009, em 
articulação com o Programa Nacional de Reorientação da Formação Profissional em Saúde (PRÓ-Saúde) (Costa e Borges, 2015). O PET-Saúde visa formar profissionais de saúde para o atendimento das necessidades da população brasileira, com referência no seu perfil socioepidemiológico, tendo como enfoque principal a integração ensino-serviço-comunidade (Morais et al., 2012). O PET-Saúde configura-se como estratégia para a consolidação do SUS, uma vez que exige ações intersetoriais para o fortalecimento da Atenção Primária, com ênfase na Estratégia Saúde da Família. Tal Programa foca na educação permanente de todos os atores envolvidos (profissionais da rede, estudantes e professores), e tem como principal desafio a efetivação dos preceitos das DCNs nos cursos de graduação da área da saúde (Cyrino et al., 2012; Haddad et al., 2012).

Um dos desafios sinalizados por uma experiência complexa como a condução e coordenação do PET-Saúde exige ferramentas que o avaliem num âmbito abrangente e levem em consideração o investimento público da ação. Nessa perspectiva, o Exame Nacional de Avaliação de Desempenho dos Estudantes (Enade), um dos componentes do Sistema Nacional de Avaliação da Educação Superior (Sinaes), tem como possibilidade trazer elementos avaliativos relativos à participação do aluno no Programa, uma vez que se configura como um exame em larga escala, aplicado aos estudantes concluintes de determinada área de conhecimento.

Para Feldman e Souza (2016), o Enade é um importante instrumento de aferição e de promoção da qualidade para os cursos de graduação e para as políticas de educação superior do país. Seu objetivo é acompanhar o processo de aprendizagem e o desempenho acadêmico dos estudantes de educação superior. É composto de dois questionários: o de avaliação discente da educação superior e o dos coordenadores de curso; além disso, há uma prova e a respectiva percepção do aluno sobre ela (Brito, 2008).

É papel preponderante do Enade, segundo Noro et al. (2017), estimular a autoavaliação dos cursos, permitindo que seja compreendido como ação transformadora, que empodere os atores diretamente envolvidos (professores e alunos) e que contribua, significativamente, para a consolidação das DCNs.

O objetivo deste estudo foi identificar a contribuição do PET-Saúde na formação em enfermagem no Brasil por meio dos resultados do Enade ocorrido em 2013.

\section{O desafio de obter dados públicos no Brasil}

Trata-se de estudo observacional, de corte transversal analítico, o qual analisou o desempenho de todos os alunos concluintes dos cursos de enfermagem brasileiros que participaram do Enade 2013. O desempenho dos estudantes que fizeram parte do PET-Saúde, entre os anos de 2009 e 2013, foi compara- 
do com aqueles que não participaram do Programa, utilizando-se as médias ponderadas em formação geral, formação profissional em Saúde Coletiva.

Para acessar a base de dados relacionada aos alunos que participaram do Enade 2013, foi realizada, primeiramente, solicitação ao Serviço de Atendimento ao Pesquisador do Instituto Nacional de Estudos e Pesquisas Estatísticas Anísio Teixeira (Inep), conforme previsto em legislação específica (Brasil, 2014). Após autorização e agendamento prévio com a Diretoria de Estudos Educacionais, foram acessados os bancos de dados relativos aos resultados do Enade 2013 em ambiente seguro, garantindo-se sigilo das informações e preservação da identidade dos estudantes.

Para o linkage com a base de dados original fornecida pelo Inep, foram obtidas referências relativas aos alunos que participaram do PET-Saúde de 2009 a 2013, por meio do Portal da Transparência do governo brasileiro, iniciativa do Ministério da Transparência, Fiscalização e Controladoria-Geral da União que busca assegurar a boa e correta aplicação dos recursos públicos (Campos, Paiva e Gomes, 2013).

No referido Portal, foi possível identificar os alunos que receberam bolsa no período, o que os diferencia daqueles que não participaram do PET-Saúde. Para a composição final do banco de dados, foi realizado um processo de fusão dos dados por meio do comando 'merge' do programa SPSS, disponibilizado na própria base de dados do Inep.

Todos os dados cedidos aos investigadores foram devidamente auditados por profissionais do Inep, garantindo, dessa forma, que as informações fornecidas não permitissem identificação quer dos estudantes participantes da Enade 2013, quer das IESs, conforme preconizado nas normas legais do Sinaes.

A prova do Enade é composta por 40 questões, sendo 10 de formação geral e 30 de conteúdos específicos, elaboradas para avaliar as habilidades acadêmicas, as competências profissionais e conhecimentos básicos de cada área, assim como questões transdisciplinares (Brasil, 2013).

As questões de formação geral são compostas por temas relevantes relacionados ao compromisso social, comportamento ético, espírito científico, humanístico e reflexivo, capacidade de análise crítica e integrada à realidade e a capacidade de socializar o conhecimento em vários contextos (Ristoff, 2014).

As questões de conteúdo específico são baseadas nas competências e habilidades específicas fornecidas pelas DCNs e foram classificadas, neste estudo, como formação profissional (questões diretamente relacionadas à prática da enfermagem) e formação em saúde coletiva (questões relacionadas diretamente à formação para o SUS).

A variável dependente deste estudo referiu-se à participação dos alunos de enfermagem no PET-Saúde. Já as variáveis independentes foram relacionadas aos resultados dos alunos do Enade 2013, considerando as médias aritméticas em Formação geral, Conteúdo específico (Formação profissional e Formação em 
Saúde Coletiva) e Desempenho global, diferenciando-se as questões de múltipla escolha das questões dissertativas. Foram ainda consideradas as características das IESs relativas à categoria administrativa e à organização acadêmica.

O banco de dados foi armazenado no Microsoft Excel ${ }^{\circledR}$ e exportado para o software R versão 3.1.117, e a análise estatística foi realizada no programa SPSS, versão 2.0. A análise estatística descritiva dos dados foi realizada com a finalidade de identificar a inserção dos concluintes dos cursos de enfermagem no Brasil, participantes ou não do PET-Saúde, segundo IESs, considerando a categoria administrativa e a organização acadêmica. Num segundo momento, foi desenvolvida análise inferencial dos dados, buscando as associações entre a participação no PET-Saúde e o desempenho do aluno no curso. Para isso, foram comparadas as médias no grupo de questões que representava cada um dos segmentos (Formação geral, Formação profissional e Formação em Saúde Coletiva e Desempenho global) por meio da análise de variância (anova), considerando-se um nível de significância de $\mathrm{p}<0,05$.

Esta pesquisa foi submetida ao Comitê de Ética em Pesquisa do Hospital Onofre Lopes - UFRN (CEP-HUOL) sob CAAE 55838816.7.000.5292, de acordo com os princípios estabelecidos na Resolução n. 466/2012 do Conselho Nacional de Saúde e aprovado pelo parecer 1.557.954, de 24 de maio de 2016.

\section{A importância da Enfermagem no SUS}

A amostra do presente estudo foi composta por 30.289 alunos concluintes de todos os cursos de enfermagem vinculados ao Enade no ano de 2013, considerando todas as IESs brasileiras. Do total, 876 alunos participaram do PETSaúde, entre os anos de 2009 e 2013. A distribuição dos alunos envolvidos no Enade 2013 e dos participantes do PET-Saúde, por categoria administrativa e organização acadêmica, encontra-se na Tabela 1 .

\section{Tabela 1}

Distribuição dos alunos de enfermagem que participaram do Enade 2013 e do PET-Saúde por categoria administrativa e organização acadêmica

\begin{tabular}{|c|c|c|c|c|}
\hline \multirow{2}{*}{ Instituição de Educação Superior } & \multicolumn{2}{|c|}{$\begin{array}{l}\text { Alunos enfermagem* } \\
\qquad(\mathrm{n}=29.413)\end{array}$} & \multicolumn{2}{|c|}{$\begin{array}{l}\text { PET-Saúde*** } \\
(\mathrm{n}=876)\end{array}$} \\
\hline & $\mathrm{n}$ & $\%$ & $\mathrm{n}$ & $\%$ \\
\hline \multicolumn{5}{|l|}{ Categoria administrativa } \\
\hline Pública & 4.353 & 14,8 & 752 & 85,8 \\
\hline Privada & 25.060 & 85,2 & 124 & 14,2 \\
\hline \multicolumn{5}{|l|}{ Organização acadêmica } \\
\hline Universidade & 11.039 & 37,5 & 836 & 95,4 \\
\hline Centro Universitário & 5.139 & 17,5 & 4 & 0,5 \\
\hline Faculdade & 13.235 & 45,0 & 36 & 4,1 \\
\hline
\end{tabular}


Observa-se, claramente, o grande predomínio de instituições privadas na formação em Enfermagem, assim como acontece na grande maioria dos cursos de graduação na área da saúde no Brasil.

\section{O PET-Saúde como diferencial na formação do enfermeiro}

O principal objetivo do presente estudo foi identificar se havia diferença de desempenho entre os alunos que participaram do PET-Saúde com aqueles que não fizeram parte do Programa. Para isso, a prova do Enade foi dividida em segmentos, de acordo com sua aplicação, em Formação geral, Formação específica e em Saúde Coletiva. A Tabela 2 apresenta os resultados, considerando os diferentes tipos de IESs, quer pela sua organização acadêmica, quer por sua categoria administrativa.

\section{Tabela 2}

Média nos segmentos Formação geral, Formação específica, Saúde Coletiva e Desempenho Global dos alunos PET Saúde e não PET dos cursos de enfermagem, por tipo de instituição de educação superior, Enade 2013

\begin{tabular}{|c|c|c|c|c|}
\hline Tipo de IES & PET-Saúde & Não PET & Anova & $\neq$ \\
\hline \multicolumn{5}{|l|}{ Todas as IES } \\
\hline Formação geral & 49,6945 & 43,5095 & $<0,001$ & 6,185 \\
\hline Formação específica & 65,1872 & 51,5705 & $<0,001$ & 13,6167 \\
\hline Saúde Coletiva & 63,3165 & 47,6210 & $<0,001$ & 15,6955 \\
\hline Desempenho global & 61,3262 & 49,5676 & $<0,001$ & 11,7586 \\
\hline \multicolumn{5}{|l|}{ Universidades } \\
\hline Formação geral & 49,6091 & 44,667 & $<0,001$ & 4,9421 \\
\hline Formação específica & 65,1227 & 54,4392 & $<0,001$ & 10,6835 \\
\hline Saúde Coletiva & 63,0654 & 51,1559 & $<0,001$ & 11,9095 \\
\hline Desempenho global & 61,2567 & 52,0083 & $<0,001$ & 9,2493 \\
\hline \multicolumn{5}{|l|}{ Públicas } \\
\hline Formação geral & 49,7464 & 48,6917 & 0,112 & 1,0547 \\
\hline Formação específica & 65,6508 & 61,7327 & $<0,001$ & 3,9181 \\
\hline Saúde Coletiva & 63,5628 & 58,4623 & $<0,001$ & 5,1005 \\
\hline Desempenho global & 61,6866 & 58,4850 & $<0,001$ & 3,2016 \\
\hline \multicolumn{5}{|l|}{ Privadas } \\
\hline Formação geral & 49,3891 & 42,5892 & $<0,001$ & 6,7999 \\
\hline Formação específica & 62,4563 & 49,7659 & $<0,001$ & 12,6904 \\
\hline Saúde Coletiva & 61,8662 & 45,6964 & $<0,001$ & 16,1698 \\
\hline Desempenho global & 59,2034 & 47,9842 & $<0,001$ & 11,2192 \\
\hline
\end{tabular}

Fonte: Inep, 2016.

IES: Instituição de educação superior.

Em todos os segmentos, observa-se melhor desempenho dos alunos que participaram do PET-Saúde em relação aos que não fizeram parte, com diferença estatisticamente significativa, exceto no caso do segmento Formação geral nas instituições públicas, no qual o melhor desempenho não apresenta diferença 
estatisticamente significativa (Anova $=0,112$ ). Observa-se que, entre todos os segmentos, a Saúde Coletiva foi a que apresentou maior diferença entre as médias dos alunos envolvidos no PET-Saúde (média = 63,3165), quando comparadas às dos alunos que não participaram do Programa (média $=47,6210$ ).

Considerando ser importante a comparação relacionada ao desempenho dos alunos participantes do PET-Saúde em diferentes tipos de instituição, buscou-se observar o desempenho dos alunos segundo sua inserção em instituições públicas ou privadas. Os resultados estão na Tabela 3.

\section{Tabela 3}

Média nos segmentos Formação geral, Formação específica, Saúde Coletiva e Desempenho global em instituições públicas e privadas pela participação ou não no PET-Saúde dos cursos de enfermagem, Enade 2013.

\begin{tabular}{|c|c|c|c|c|}
\hline Aluno & Pública & Privada & Anova & $\neq$ \\
\hline \multicolumn{5}{|l|}{ PET-Saúde } \\
\hline Formação geral & 49,7464 & 49,3891 & 0,837 & 0,3573 \\
\hline Formação específica & 65,6508 & 62,4563 & 0,051 & 3,1945 \\
\hline Saúde Coletiva & 63,5628 & 61,8662 & 0,416 & 1,6966 \\
\hline Desempenho global & 61,6866 & 59,2034 & 0,099 & 2,4832 \\
\hline \multicolumn{5}{|l|}{ Não PET } \\
\hline Formação geral & 48,6917 & 42,5892 & $<0,001$ & 6,1025 \\
\hline Formação específica & 61,7327 & 49,7659 & $<0,001$ & 11,9668 \\
\hline Saúde Coletiva & 58,4623 & 45,6964 & $<0,001$ & 12,7659 \\
\hline Desempenho global & 58,4850 & 47,9842 & $<0,001$ & 10,5008 \\
\hline
\end{tabular}

Enquanto o Desempenho global dos alunos que não participaram do PET-Saúde é melhor nas instituições públicas (média $=58,485$ ) do que nas instituições privadas (média $=47,9842$ ), o mesmo não se observa em relação àqueles que fizeram parte do PET-Saúde. Nota-se que não existe diferença estatisticamente significativa em relação às médias no Desempenho global entre aqueles que se formaram em instituições públicas (média $=61,6866$ ) com os formados em instituições privadas (média $=59,2034$ ). Situação idêntica acontece em relação à Formação geral, Formação específica e Saúde Coletiva.

\section{A formação para o SUS consolidada pelo PET-Saúde}

A formação em todos os cursos na área da saúde, em princípio, deveria ter como pauta prioritária a eleição do SUS como lócus obrigatório para sua ordenação. Tal prerrogativa vem fortemente alicerçada nas conquistas proporcionadas pela constituição brasileira de 1988 - a qual propõe saúde como direito humano fundamental e expressão de cidadania nacional -, alcançadas por um histórico processo de lutas arregimentadas pelo Movimento da Reforma Sanitária (Gusmão, Ceccim e Drachler, 2015).

$\mathrm{Na}$ área da Enfermagem, há diversas sinalizações de avanços promovidos tanto pela formação de trabalhadores com foco nas necessidades de saúde 
da população quanto pela produção de conhecimentos inovadores e úteis para a sociedade (Dias et al., 2016; Brehmer e Ramos, 2016). Entretanto, são também identificadas lacunas na formação vinculadas à interdisciplinaridade e à transversalidade de conteúdos relacionados ao SUS (Winters, Prado e Heidemann, 2016).

Na percepção de Brehmer e Ramos (2017), a enfermagem atuou como pioneira entre os cursos da área da saúde para a promoção de mudanças no processo de formação profissional. Essas transformações foram alcançadas, especialmente, desde a promulgação das DCNs dos cursos de graduação, em 2011. Para alcance dessa proposta, ainda segundo as autoras, vários cursos propuseram currículos estreitamente vinculados ao SUS, com enfoque privilegiado na atenção primária à saúde e com equipes de saúde efetivamente relacionadas ao território das áreas adscritas às unidades de saúde.

Entre as diversas iniciativas para a efetivação da formação vinculada ao SUS, é possível que o PET-Saúde seja aquela que mais tem visibilidade na academia e nos serviços, considerando a articulação entre alunos, professores e profissionais (preceptores) do SUS. Reforçando essa perspectiva, vasta literatura científica foi produzida com base em estudos desenvolvidos no âmbito do Programa (Morais et al., 2012; Santos, Almeida e Reis, 2013; Pinto et al., 2013; Freitas et al., 2013; Santos et al., 2015; Costa et al., 2015). A quase totalidade desses estudos sinaliza os possíveis avanços proporcionados, desde 2009, pelo PET-Saúde. Esses estudos abordam, grande parte das vezes, relatos de situações específicas de determinada instituição de educação superior, utilizando enfoques identificados com a divulgação científica de experiências pontuais do Programa.

Assim como em outros (Dias et al. 2016; Farias-Santos e Noro, 2017), buscou-se, com o presente estudo, uma abordagem mais ampla na perspectiva de avaliar o impacto do PET-Saúde na formação do concluinte com base nos resultados do Enade. Tal estratégia foi utilizada por se considerar o exame um mecanismo para avaliar o desempenho dos estudantes em relação às habilidades e competências previstas nas DCNs. Buscou-se, na realidade, maior possibilidade de generalização, uma vez que o público-alvo do estudo foi composto por todos os concluintes de Enfermagem do país, independentemente da organização acadêmica ou da categoria administrativa da IES.

Por sua concepção, o Enade busca avaliar o desempenho dos estudantes em relação aos conteúdos programáticos previstos nas DCNs dos cursos de graduação. Do mesmo modo, almeja o desenvolvimento de competências e habilidades necessárias ao aprofundamento da formação geral e profissional e a melhoria do nível de atualização dos estudantes com relação à realidade brasileira e mundial, e deve servir como referência para as revisões curriculares e suas possíveis adequações (Brasil, 2004). Nesse contexto, ressalta-se que o Enade é uma avaliação que tem por base não o perfil do concluinte, mas o 
perfil da formação propiciada pelo curso (Ristoff e Limana, 2007). Desse modo, o desempenho dos alunos mostra-se como um ótimo referencial para identificar o quanto os currículos dos cursos de Enfermagem procuraram direcionar sua formação para as DCNs, em especial para o SUS, o qual é preconizado como cenário ideal para aprendizagem a partir da realidade social e dos serviços de saúde. No presente estudo, essa perspectiva encontra-se potencializada ao buscar elementos que diferenciem os alunos que participaram do PET-Saúde daqueles que não fizeram parte do referido Programa.

Dentre os resultados apontados, é importante sinalizar que, aproximadamente, $85 \%$ dos alunos concluintes dos cursos de enfermagem no ano de 2013 estavam vinculados às IES privadas. Esse percentual sinaliza a hegemonia do modelo privatista da educação superior iniciado nos anos 1990 no Brasil. Observa-se que o processo de avaliação externa visa ao cumprimento do papel social de qualquer curso da área da saúde e à necessidade de coerência de seu projeto pedagógico com as DCNs. Assim, é essencial que esse processo, o qual regula o funcionamento dos cursos de graduação, garanta a efetiva formação desses alunos na lógica do acesso e cobertura universal da saúde (Zug et al., 2016), buscando coerência com a formação no SUS. Considerando a abrangência dos critérios observados ao longo do processo de avaliação externa, maior atenção deveria ser dispensada pelos avaliadores aos Contratos Organizativos de Ação Pública de Ensino e Saúde (Brasil, 2015), previstos como requisitos legais e normativos no Instrumento de Avaliação de Cursos de Graduação presencial e à distância (Brasil, 2016).

Quanto aos resultados relacionados ao desempenho dos alunos, FariasSantos e Noro (2017) também verificaram melhor desempenho dos alunos que participaram do PET-Saúde em relação aos demais. Essa constatação independe da organização acadêmica ou categoria administrativa, com diferença estatisticamente significativa em todos os segmentos, exceto na formação geral nas instituições públicas.

Também deve ser levado em consideração que o PET-Saúde proporciona avanços na formação em saúde, tanto pela atuação diferenciada do docente como pela contribuição para a qualificação dos profissionais inseridos nos serviços de saúde (Fonseca e Junqueira, 2014; Alves et al., 2015).

Entre todos os segmentos, a Saúde Coletiva foi a que apresentou maior diferença entre as médias dos alunos que participaram do PET-Saúde, quando comparadas às dos alunos que não fizeram parte do Programa. Tal resultado indica o efetivo impacto do PET-Saúde na formação, alinhando-se à percepção dos alunos, os quais atribuem ao Programa contribuições na formação interprofissional, na promoção da saúde e nas atividades de prevenção de agravos, que fortalecem a possibilidade de aprendizado na atenção primária (Pinto et al., 2013; Caldas et al., 2012; Kovaleski et al., 2016). 
Uma contribuição inequívoca dos resultados apresentados é a busca imperiosa, conforme outros estudos (Alves et al., 2015 ; Kovaleski et al., 2016), pelo protagonismo do SUS na construção de currículos. Dessa forma, a construção do conhecimento se inicia na plena relação ensino-serviço, empoderando os profissionais e valorizando a capacidade docente em incentivar seu aluno na conquista de melhores condições de saúde (e vida) para a população.

melhores condições de saúde (e vida) para a populacao.Vale ressaltar que, para a efetiva inclusão do SUS nos currículos, são necessárias adequações que permitam transformar as unidades de saúde em lócus privilegiados de aprendizado, constituídos por instalações físicas que permitam, além da assistência adequada à população, desenvolvimento de metodologias ativas de aprendizagem (Gonçalves et al., 2015).

Outra contribuição do presente estudo, o qual reforça a perspectiva do PET-Saúde como estratégia diferenciada, refere-se à análise dos alunos que participaram no Programa em IESs de diferentes categorias administrativas. Observa-se que o desempenho do aluno do PET-Saúde refere-se muito mais ao Programa do que ao tipo de estabelecimento ao qual o aluno está matriculado, visto que não se observa diferença no desempenho desses alunos no Enade, independente de se tratar de IES pública ou privada.

Fica claro, no presente estudo, o papel diferenciado que o poder púbico deve desempenhar na perspectiva de fazer valer preceitos estabelecidos nas DCNs dos cursos de graduação em saúde, em especial, na formação vinculada ao SUS. Estratégias como o PET-Saúde deveriam ser universalizadas, de forma a garantir que pesquisa, ensino e extensão pudessem contribuir com a responsabilidade social das IESs na transformação dos alunos em reais defensores de um sistema de saúde público e vinculado às expectativas da população.

Entretanto, o atual cenário prevê a redução de recursos públicos para a área da saúde, assim como para outras áreas estratégicas que dependem de uma política pública efetivamente vinculada aos preceitos constitucionais. Na compreensão de Bravo, Pelaez e Pinheiro (2018), o congelamento de recursos orçamentários por vinte anos para as políticas sociais desempenha papel central no estímulo à privatização da saúde e funciona como mecanismo de 'contrarreforma', considerando a aceleração do desmonte das políticas públicas e universais. Ainda, segundo os autores, essas medidas ganham destaque ao serem observados os retrocessos recentes na política de saúde mental, nas mudanças na Política Nacional de Atenção Básica e na articulação com o setor privado na perspectiva da substituição do SUS por planos de saúde ditos 'populares'.

Para Bahia et al. (2016), a proposta de expansão de planos de saúde 'populares' expressa a redução de direitos sociais previstos em lei, o que contribuirá, sobremaneira, para o aprofundamento das iniquidades no acesso à saúde no Brasil. 
Uma das limitações desta pesquisa refere-se ao fato de que seus resultados são decorrentes do Enade, exame que não tem impacto, diretamente, na graduação do aluno, nem em sua futura inserção no mercado de trabalho, uma vez que o desempenho do discente é disponibilizado somente para ele. Outra limitação refere-se ao questionamento do Enade como estratégia efetivamente capaz de medir o conhecimento do concluinte, por se tratar, basicamente, de um exame, perspectiva sinalizada em alguns estudos que analisam sua característica psicométrica (Primi, Hutz e Silva, 2011). Apesar dessas limitações, o Enade deve corresponder efetivamente aos conhecimentos e habilidades adquiridos pelos estudantes numa perspectiva global, uma vez que se trata de importante referência para o Sinaes (Wainer e Melguizo, 2018). Entretanto, apesar dessas limitações, para Wainer e Melguizo (2018), o Enade, numa perspectiva global, deve corresponder, de forma efetiva, à parte dos conhecimentos e habilidades adquiridos pelos estudantes, uma vez que se trata de importante referência para o Sinaes.

\section{Considerações finais}

O PET-Saúde, investimento compartilhado entre o Ministério da Saúde e o da Educação, tem como principal eixo a reformulação dos currículos dos cursos da área da saúde coerente com as DCNs. Tem como elemento central a articulação entre alunos e professores de cursos de graduação e profissionais de saúde do SUS (preceptores), permitindo a efetiva inserção da academia nos serviços de saúde.

Além das evidências anteriores, os resultados do presente estudo revelam de forma contundente que o PET-Saúde tem potencial diferenciado na compreensão da necessidade da formação no SUS, envolvendo conhecimentos de Saúde Coletiva e da área profissional.

É, portanto, fundamental que iniciativas como o PET-Saúde sejam fortalecidas pelo poder público e prevejam formas concretas de garantir a construção de currículos centrados no SUS para os cursos que participam do Programa, considerando o estímulo a todos os atores desse processo, assim como a proposição de melhores respostas às necessidades de saúde da população.

Deve-se lembrar, entretanto, que a atual política econômica em muito contribui para dificultar os principais avanços observados neste estudo. É urgente que, para aqueles que acreditam no SUS como o modelo mais adequado para o exercício dos direitos da população na área da saúde, algumas ações que envolvam a sociedade organizada e as parcelas mais progressistas do mundo acadêmico sejam articuladas fundamentadas em todos os avanços alcançados nos últimos trinta anos de uma ainda incipiente política pública de saúde. 


\section{Colaboradores}

Luiz Roberto Augusto Noro participou da concepção do projeto, da redação do projeto de pesquisa, revisão crítica da literatura, planejamento do estudo, coleta e interpretação dos dados e revisão crítica do manuscrito. José Luis Medina Moya participou da concepção do projeto, revisão crítica da literatura, planejamento do estudo e revisão crítica do manuscrito. Não há conflito de interesses.

\section{Financiamento}

O presente trabalho foi realizado com apoio da Coordenação de Aperfeiçoamento de Pessoal de Nível Superior - Brasil (CAPES) - Código de Financiamento 001.

\section{EL PET-SALUD COMO ORIENTADOR DE LA FORMACIÓN EN ENFERMERÍA PARA EL SISTEMA ÚNICO DE SALUD}

Resumen La salud de calidad y de acceso universal es un derecho que debe ser garantizado a partir de políticas públicas, financiación compatible y la adecuada formación de los profesionales de salud. Una de las estrategias identificadas para alcanzar este desafío es el Programa de Educación para el Trabajo en Salud, que busca promover la interacción activa de alumnos y profesores con los servicios de salud. El objetivo de este estudio fue utilizar los resultados del Examen Nacional de Rendimiento de los Estudiantes de las carreras de Enfermería del 2013 para comparar el rendimiento de aquellos que participaron de dicho Programa con el de aquellos que no formaron parte del mismo. Se trata de estudio observacional, de corte transversal analítico, realizado en el período de febrero a junio del 2017. La investigación analizó el rendimiento a partir de las medias ponderadas en formación general, formación profesional y salud pública. La muestra del estudio consistió en 30.289 estudiantes, de los cuáles 876 estaban vinculados al Programa de Educación para el Trabajo en Salud. Los alumnos involucrados con el Programa tuvieron un rendimiento superior en todos los segmentos, con diferencia estadísticamente significativa ( $p<0,001)$ en comparación con aquellos que no participaron del mismo. Los resultados indican que el Programa en cuestión tiene potencial para comprender la necesidad de la formación en el Sistema Único de Salud, incluyendo conocimientos de Salud Pública y del área profesional, debiendo servir como elemento orientador de la construcción de los programas de las carreras de Enfermería.

Palabras clave Sistema Único de Salud; evaluación educativa; enfermería. 


\section{Referências}

ALVES, Cláudia R. L. et al. Repercussões do Programa de Educação pelo Trabalho para a Saúde (PET-Saúde) na reforma curricular de escolas médicas participantes do Programa de Incentivos às Mudanças Curriculares dos cursos de Medicina (PROMED). Revista Brasileira de Educação Médica, Brasília, v. 39, n. 4, p. 527-536, 2015.

BAHIA, Ligia et al. Planos privados de saúde com coberturas restritas: atualização da agenda privatizante no contexto de crise política e econômica no Brasil. Cadernos de Saúde Pública, Rio de Janeiro, v. 32, n. 12, 2016.

BRASIL. Ministério da Saúde. Portaria Interministerial 1.124, de 4 de agosto de 2015. Institui as diretrizes para a celebração dos Contratos Organizativos de Ação Pública Ensino-Saúde (COAPES), para o fortalecimento da integração entre ensino, serviços e comunidade no âmbito do Sistema Único de Saúde (SUS). Diário Oficial [da] República Federativa do Brasil, Poder Executivo, Brasília: MS, 2015. Disponível em: <http://www.crub.org.br/blog/portariainterministerial-no-1-124-de-4-de-agostode-2015/> Acesso em: 15 maio 2017.

BRASIL. Lei $\mathrm{n}^{\circ}$ 10.861, de 14 de abril de 2004. Institui o Sistema Nacional de Avaliação da Educação Superior-SINAES e dá outras providências. Diário Oficial [da] República Federativa do Brasil, Poder Executivo, Brasília: MS, 2004. Disponível em: <http://www.planalto.gov.br/ ccivil_03/_Ato2004-2006/2004/Lei/L10.861. htm> Acesso em: 15 abr. 2017.

BRASIL. Ministério da Educação. Instituto Nacional de Estudos e Pesquisas Educacionais Anísio Teixeira. Portaria INEP - n. 467, de 19 de setembro de 2014. Brasília: Ministério da Educação, 2014. Disponível em: <http:// www.lex.com.br/legis_25984628_PORTARIA_ N_467_DE_19_DE_SETEMBRO_DE_2014. aspx> Acesso em: 10 mar. 2017.
BRASIL. Ministério da Educação. Instituto Nacional de Estudos e Pesquisas Educacionais Anísio Teixeira - INEP. Manual do ENADE. Diário Oficial [da] República Federativa do Brasil, Poder Executivo, Brasília: Ministério da Educação, 2013. Disponível em: <http:// www.planalto.gov.br/ccivil_03/leis/L8080. htm>. Acesso em: 10 jul. 2018.

BRASIL. Ministério da Educação. Instituto Nacional de Estudos e Pesquisas Educacionais Anísio Teixeira - INEP. Diretoria de Avaliação da Educação Superior- DAES. Sistema Nacional de Avaliação da Educação Superior- SINAES. Instrumento de Avaliação de Cursos de Graduação presencial e à distância. Brasília: Ministério da Educação, 2016. Disponível em: <http:// download.inep.gov.br/educacao_superior/ avaliacao_cursos_graduacao/instrumentos/2016/instrumento_2016.pdf >. Acesso em: 12 fev. 2017.

BRAVO, Maria I. S.; PELAEZ, Elaine J.; PINHEIRO, Wladimir N. As contrarreformas na política de saúde do governo Temer. Argumentum Debate, Vitória, Espírito Santo, v. 10, n. 1, p. 9-23, 2018.

BREHMER, Laura C. F.; RAMOS, Flávia R. S. Experiências do Programa de Reorientação da Formação Profissional na Enfermagem: avanços e desafios. Texto e Contexto Enfermagem, Florianópolis, v. 26, n. 2, 2017.

BREHMER, Laura C. F.; RAMOS, Flávia R. S. O modelo de atenção à saúde na formação em enfermagem: experiências e percepções. Interface: Comunicação, Saúde e Educação, Botucatu, SP, v. 20, n. 56, p. 135-145, 2016.

BRITO, Márcia R. F. SINAES e o ENADE: da concepção à implantação. Avaliação: Revista da Avaliação da Educação Superior, Sorocaba, SP, v. 13, n. 3, p. 841-850, 2008.

CALDAS, Juliana B. et al. A percepção de alunos quanto ao Programa de Educação pelo 
Trabalho para a Saúde (PET-Saúde). Revista Brasileira de Educação Médica, Brasília, v. 36, p. 33-41, 2012. Suplemento 1.

CAMPOS, Rosana; PAIVA, Denise; GOMES, Suely. Gestão da informação pública: um estudo sobre o Portal Transparência Goiás. Sociedade e Estado, Brasília, v. 28, n. 2, p. 393-417, 2013.

COSTA, Marcelo V. et al. Pró-Saúde e PET-Saúde como espaços de educação interprofissional. Interface: Comunicação, Saúde e Educação, Botucatu, v. 19, p. 709-720, 2015. Suplemento 1.

COSTA, Marcelo V.; BORGES, Flávio A. O PróPET-Saúde frente aos desafios do processo de formação profissional em saúde. Interface: Comunicação, Saúde e Educação, Botucatu, v. 19, p. 753-763, 2015. Suplementol.

CYRINO, Eliana G. et al. Ensino e pesquisa na estratégia de saúde da família: o PET-Saúde da FMB/UNESP. Revista Brasileira de Educação Médica, Brasília, v. 36, n. suplemento 1, p. 92-101, 2012.

DIAS, Maria A. S. et al. Caracterização das graduações em enfermagem segundo Exame Nacional de Desempenho de Estudantes. Revista Brasileira de Enfermagem, Brasília, v. 68, n. 2, p. 375-381, 2016.

FARIAS-SANTOS, Bárbara C. S.; NORO, Luiz R. A. PET-Saúde como indutor da formação profissional para o Sistema Único de Saúde. Ciência e Saúde Coletiva, Rio de Janeiro, v. 22, n. 3, p. 997-1.004, 2017.

FELDMAN Taise; SOUZA, Osmar. A governamentalidade e o Exame Nacional de Desempenho de Estudantes - ENADE. Avaliação: Revista da Avaliação da Educação Superior, Sorocaba, v. 21, n. 3, p. 1.017-1.032, 2016.

FONSECA, Graciela S.; JUNQUEIRA, Simone R. Programa de Educação pelo Trabalho para a Saúde da Universidade de São Paulo (Campus Capital): o olhar dos tutores. Ciência e Saúde Coletiva, Rio de Janeiro, v. 19, n. 4, p. 1.151-1.162, 2014.
FREITAS, Paula H. et al. Repercussões do PETSaúde na formação de estudantes da área da saúde. Escola Anna Nery, Rio de janeiro, v. 17, n. 3, p. 496-504, 2013.

GONÇALVES, Rita C. R. et al. Nós em rede: vivências da parceria ensino-serviço produzidas pelo Programa de Educação pelo Trabalho para a Saúde. Interface: Comunicação, Saúde e Educação, Botucatu, v. 19, p. 903-912, 2015. Suplemento 1.

GUSMÃO, Renata C.; CECCIM, Ricardo B.; DRACHLER, Maria L. Tematizar el impacto en la educación en el trabajo para la salud: abrir gavetas, enunciar preguntas, escribir. Interface: Comunicação, Saúde e Educação, Interface: Comunicação, Saúde e Educação, Botucatu, v. 19, p. 695-707, 2015. Suplementol.

HADDAD, Ana E. A enfermagem e a política nacional de formação dos profissionais de saúde para o SUS. Revista da Escola de Enfermagem da USP, São Paulo, v. 45, n. especial 2, p. 1.803-1.809, 2011.

HADDAD, Ana E. et al. Pró-Saúde e PETSaúde: a construção da política brasileira de reorientação da formação profissional em saúde. Revista Brasileira de Educação Médica, Brasília, v. 36, p. 3-4, 2012. Suplemento 1.

KOVALESKI, Douglas F. et al. Trajetória do Pró-PET: saúde da família no cotidiano da promoção da saúde. Revista Brasileira de Educação Médica, Brasília, v. 40, n. 4, p. 765-771, 2016.

MORAIS, Fátima R. R et al. A importância do PET-Saúde para a formação acadêmica do enfermeiro. Trabalho, Educação e Saúde, Rio de Janeiro, v. 10, n. 3, p. 541-551, 2012.

NORO, Luiz R. A. et al. Relação entre conteúdos das disciplinas de curso de odontologia e os ENADE 2004/2010. Avaliação: Revista da Avaliação da Educação Superior, Sorocaba, v. 22, n. 1, p. 125-139, 2017.

PEREIRA, Ingrid D. F.; LAGES Itamar. Diretrizes curriculares para a formação de profissionais 
de saúde: competências ou práxis?. Trabalho, Educação e Saúde, Rio de Janeiro, v. 11, n. 2, p. 319-338, 2013.

PIERANTONI, Celia R.; MAGNAGO, Carinne. Tendências das graduações em Saúde no Brasil: análise da oferta no contexto do Mercosul. Divulgação em Saúde para Debate, Rio de Janeiro, n. 57, p. 30-43, 2017.

PINTO, Anna C. M. et al. Percepção dos alunos de uma universidade pública sobre o Programa de Educação pelo Trabalho para a Saúde. Ciência e Saúde Coletiva, Rio de Janeiro, v. 18, n. 8, p. 2210-2210, 2013.

PRIMI, Ricardo; HUTZ, Cláudio S.; SILVA, Marjorie C. R. A prova do ENADE de psicologia 2006: concepção, construção e análise psicométrica da prova. Interamerican Journal of Psychological Assessment, Glen Allen, v. 10, n. 3, p. 271-294, 2011.

RISTOFF, Dilvo. O novo perfil do campus brasileiro: uma análise do perfil socioeconômico do estudante de graduação. Avaliação: Revista da Avaliação da Educação Superior, Sorocaba, v. 19, n. 3, p. 723-747, 2014.

RISTOFF, Dilvo; LIMANA Amir. O ENADE como parte da avaliação da educação superior. INEP, 2007. Disponível em: <http;//3em.ubi. pt/o_enade.pdf>. Acesso em: 22 maio 2018.
SANTOS, Débora S.; ALMEIDA, Lenira M. W. S.; REIS, Renata K. Programa de Educação pelo Trabalho para Saúde: experiência de transformação do ensino e prática de enfermagem. Revista da Escola de Enfermagem da USP, São Paulo, v. 47, n. 6, p. 1.431-1.436, 2013.

SANTOS, Marize M. et al. PET-Saúde: uma experiência potencialmente transformadora no ensino de graduação. Interface: Comunicação, Saúde e Educação, Botucatu, SP, v. 19, p. 893-901, 2015. Suplemento 1.

WAINER, Jacques; MELGUIZO Tatiana. Políticas de inclusão no ensino superior: avaliação do desempenho dos alunos baseado no ENADE de 2012 a 2014. Educação e Pesquisa, São Paulo, v. 44, 2018.

WINTERS, Joanara R. F.; PRADO Marta L.; HEIDEMANN, Ivonete T. S. B. A formação em enfermagem orientada aos princípios do Sistema Único de Saúde: percepção dos formandos. Escola Anna Nery, Rio de Janeiro, v. 20, n. 2, p. 248-253, 2016.

ZUG, Keri E. et al.Enfermagem de prática avançada na América Latina e no Caribe: regulação, educação e prática. Revista Latino-Americana de Enfermagem, Ribeirão Preto, SP, v. 24, 2016. 